phys. stat. sol. (b) 228, No. 1, 121-124 (2001)

\title{
Two-Component Photoluminescence Decay in InGaN/GaN Multiple Quantum Well Structures
}

\author{
Shin-Wei Feng (a), Yung-Chen Cheng (a), Chi-Chin Liao (a), Yi-Yin Chung (a), \\ Chin-Wen Liu (a), Chin-Chung Yang ${ }^{1}$ ) (a), Yen-Sheng Lin (b), \\ Kung-Jeng Ma (b), and Jen-InN Chyi (c)
}

(a) Department of Electrical Engineering and Graduate Institute of Electro-Optical Engineering, National Taiwan University, 1, Roosevelt Road, Sec. 4, Taipei, Taiwan

(b) Department of Mechanical Engineering, Chung Cheng Institute of Technology, Tahsi, Taoyuan, Taiwan

(c) Department of Electrical Engineering, National Central University, Taiwan

(Received June 21, 2001; accepted August 4, 2001)

Subject classification: 68.55.Jk; 68.55.Ln; 78.55.Cr; 78.66.Fd; S7.14

\begin{abstract}
Two-component decay of time-resolved photoluminescence (TRPL) intensity in three InGaN/GaN multiple quantum well samples were observed. The first-decay component was attributed to exciton relaxation of free-carrier and localized states; the second-decay one was dominated by the relaxation of localized excitons. The second-decay lifetime was related to the extent of carrier localization or indium aggregation and phase separation. The lifetime of free-carrier states was connected with the defect density. Based on the temperature-dependent data of PL and stimulated emission (SE), the localization energies of the three samples were calibrated to show the consistent trend with the second-decay lifetime and previous material analyses.
\end{abstract}

Introduction Due to the low miscibility between InN and $\mathrm{GaN}$, indium aggregation and phase separation (formation of either $\mathrm{InN}$ or $\mathrm{GaN}$ clusters) occurred in InGaN/ GaN quantum well (QW) structures [1]. Indium-rich clusters in such a QW structure form the localized states, which may effectively trap carriers for radiative recombination [2]. In this paper, we report several novel optical properties of three InGaN/GaN QW samples. We conducted time-resolved photoluminescence (TRPL) measurements and observed two-component decay of photoluminescence intensity. We also combine the temperature-dependent data of PL and stimulated emission (SE) for calibrating the localization energy, which is regarded as the energy difference between the localized and free-carrier states (both at the ground levels). Note that excitons can exist in both localized and free-carrier states.

The samples were grown in a low-pressure metal-organic chemical vapor deposition reactor. The InGaN/GaN multiple QW samples consisted of five periods of Si-doped InGaN well with $3 \mathrm{~nm}$ in thickness. The designated indium compositions of the sample were 11,16 , and $21 \%$ (samples $\mathrm{A}, \mathrm{B}$, and $\mathrm{C}$, respectively). The Si doping concentration was $10^{18} \mathrm{~cm}^{-3}$. The barrier was $7 \mathrm{~nm} \mathrm{GaN}$. In each sample, the $\mathrm{QW}$ layers were sandwiched with a $1.5 \mu \mathrm{m} \mathrm{GaN}$ buffer layer on a sapphire substrate and a $50 \mathrm{~nm}$ GaN cap layer. The growth temperatures were 1050 and $740{ }^{\circ} \mathrm{C}$ for $\mathrm{GaN}$ and $\mathrm{InGaN}$, respectively.

We used HeCd (3.815 eV) laser and the fourth harmonic (4.661 eV) of a Q-switch Nd: YAG laser for PL and edge-mode SE measurements, recpectively. The TRPL meas-

$\left.{ }^{1}\right)$ Corresponding author; Phone: 886-2-23657624, Fax: 886-2-23652637, e-mail: ccy@cc.ee.ntu.edu.tw 


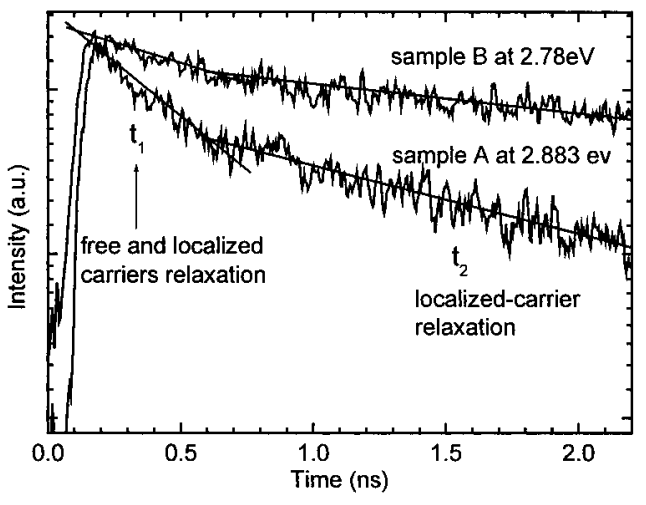

Fig. 1. Typical TRPL profiles and fitting curves

urements were performed with a Hamamatsu streak camera. Frequency-doubled optical pulses of about $100 \mathrm{fs}$ in width were generated from a mode-locked Ti:sapphire laser for excitation. The pump photon energy and power of TRPL were $3.1 \mathrm{eV}(400 \mathrm{~nm})$ and $20 \mathrm{~mW}$, respectively.

Experimental Results Two room-temperature TRPL intensity profiles of different samples at two different photon energies are shown in Fig. 1. The two-component decay in either profile can be clearly seen. Although a multiple-component decay feature in wurtzite InGaN samples was reported [3], the decay mechanism in InGaN/GaN QWs has not been fully discussed yet.

We fitted our data with two-step exponential decay to obtain the first-decay time $t_{1}$ and second-decay time $t_{2}$. The second lifetimes of the three samples are shown as functions of emission photon energy in the upper portion of Fig. 2. The decay time $t_{2}$ increases with decreasing photon energy for each of all three samples. This is a typical property of the carrier localization model [4]. The second-decay lifetimes can be regarded to describe the relaxation of localized excitons. Therefore, $t_{2}$ can be re-defined as $t_{\mathrm{L}}$, the localized carrier lifetime. Among the three samples, the increasing trend of $t_{\mathrm{L}}$ with increasing nominal indium content is consistent with, what previously has been

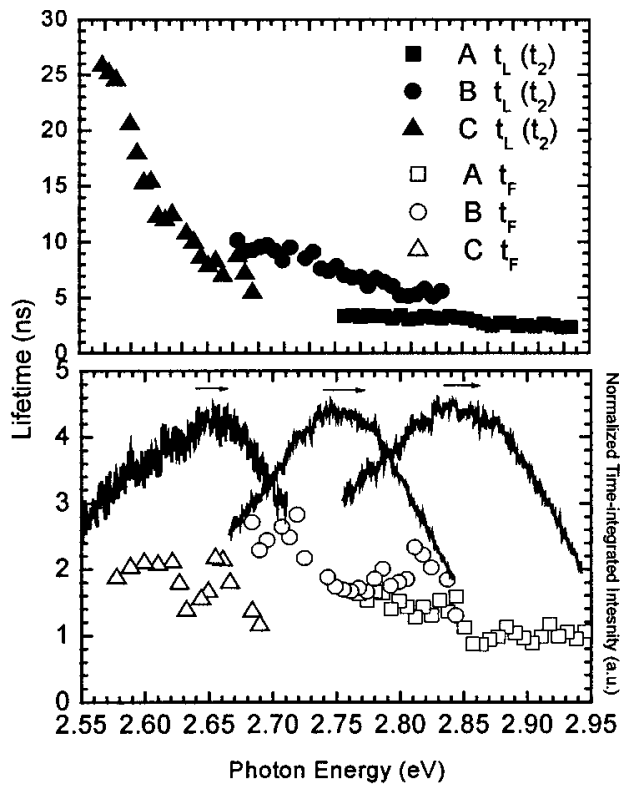
reported, that stronger carrier localization resulted in a longer lifetime [5]. On the other hand, $t_{1}$ describes the decay rate of simultaneous exciton relaxation of the free-carrier and localized states. In other words, $1 / t_{1}=1 / t_{\mathrm{F}}+1 / t_{\mathrm{L}}$, where $t_{\mathrm{F}}$ is the lifetime of free-carrier states. Thus, from the last equation we can obtain $t_{\mathrm{F}}$, which is strongly related to defect recombination. The data of $t_{\mathrm{F}}$ are shown in the lower portion of Fig. 2. Note that on the low energy side, $t_{\mathrm{F}}$ of sample $\mathrm{B}$ is larger than those of the other two samples. Hence, it

Fig. 2. Decay times $t_{\mathrm{L}}$ (top) and $t_{\mathrm{F}}$ (bottom) as functions of emission photon energy for the three samples at room temperature 


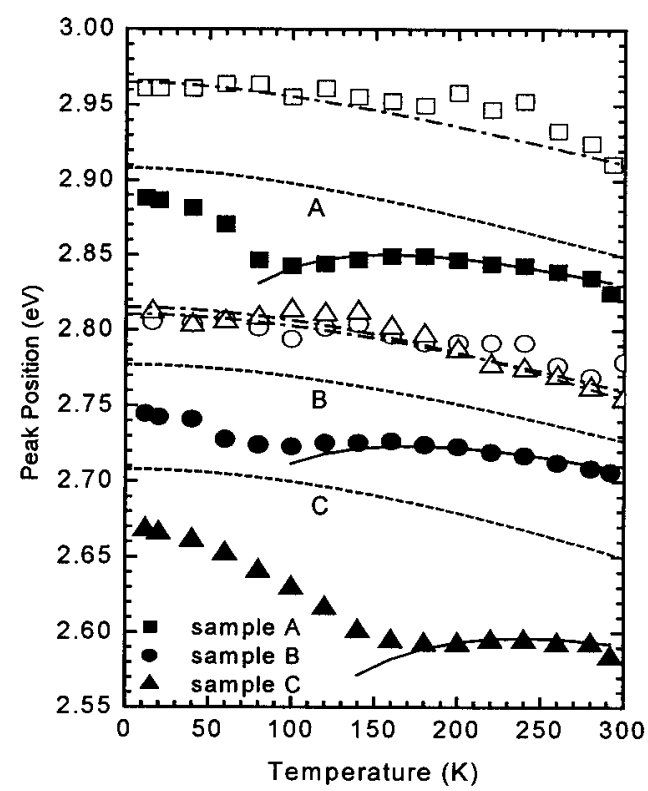

$$
E_{\mathrm{S}}(T)=E_{\mathrm{S}}(0)-\alpha T^{2} /(T+\beta) .
$$

The three dash-dotted curves stand for the fitting results of SE data with Varshni's equation. Table 1 lists the fitting values of $E_{\mathrm{S}}(0), \alpha$ and $\beta$. On the other hand, because $\mathrm{PL}$ is supposed to come from the recombination in both free-carrier and localized states, the formula for characterizing its temperature variations is given as follows [8]:

$$
E_{\mathrm{P}}(T)=E_{\mathrm{P}}(0)-\alpha T^{2} /(T+\beta)-\sigma^{2} / k_{\mathrm{B}} T .
$$

With the same values of $\alpha$ and $\beta$ for fitting the SE data, we can obtain $E_{\mathrm{P}}(0)$ and $\sigma$ by fitting the high temperature portions of the PL data in Fig. 3 (shown as the solid curves). The fitting results of $E_{\mathrm{P}}(0)$ and $\sigma$ are also listed in Table 1 . Then, keeping the same $\alpha, \beta$ and $E_{\mathrm{P}}(0)$ values in (2) and ignoring the contribution of the localized-state term, i.e., $\sigma^{2} / k_{\mathrm{B}} T$, we can reproduce the pure effect of band gap shrinkage of the freecarrier states (ground level), as shown with the short-dashed curves in Fig. 3. As a result, the energy difference between the PL peak position at the lowest temperature

Table 1

Fitting parameter values and fitting results in Fig. 3

\begin{tabular}{lccc}
\hline & sample A & sample B & sample C \\
\hline$E_{\mathrm{S}}(0)(\mathrm{eV})$ & 2.965 & 2.810 & 2.815 \\
$\alpha(\mathrm{meV} / \mathrm{K})$ & 0.37 & 0.42 & 0.67 \\
$\beta(\mathrm{K})$ & 264.6 & 450 & 700.7 \\
$E_{\mathrm{P}}(0)(\mathrm{eV})$ & 2.908 & 2.777 & 2.708 \\
$\sigma(\mathrm{meV})$ & 22 & 22.3 & 38.24 \\
localization energy $E_{\mathrm{L}}(\mathrm{meV})$ & 21.3 & 35.4 & 41.27 \\
distribution energy $E_{\mathrm{D}}(\mathrm{meV})$ & 57 & 43 & 107 \\
\hline
\end{tabular}


and the intercepted point of the short-dashed curve at $0 \mathrm{~K}$ (i.e., $\left.E_{\mathrm{P}}(0)\right)$ roughly corresponds to the energy difference between the localized and free-carrier states (both at the ground levels), i.e., the localization energy, $E_{\mathrm{L}}$. The calibrated values of $E_{\mathrm{L}}$ are shown in Table 1 . We also listed the values of distribution energy, $E_{\mathrm{D}}$, defined as the difference between the SE peak energy and the presumably ground level of free-carrier states. In other words, $E_{\mathrm{D}}=E_{\mathrm{S}}(0)-E_{\mathrm{P}}(0)$.

Several interesting features can be observed in Table 1. The increasing trend of $\sigma$ with increasing indium content is reasonable because it is proportional to the degree of carrier localization. This trend is consistent with the high-resolution transmission electron microscopy (HRTEM) images [1]. One can see the trend that a higher nominal indium content leads to a larger localization energy. This trend again is consistent with the extent of composition fluctuation, confirmed with HRTEM and X-ray diffraction (XRD) [1]. Note that the localization energies of samples $\mathrm{B}$ and $\mathrm{C}$ are closer, compared with sample A. Nevertheless, the $\sigma$ values of samples A and B are closer, compared with sample $\mathrm{C}$. These results can be explained with the following hypotheses. The number densities of local potential minima (indium-rich clusters), which are supposed to be related to the $\sigma$ values, of samples $\mathrm{A}$ and $\mathrm{B}$ are about the same; however, the potential depths of these minima (indium composition contrasts), which are represented by the localization energy $E_{\mathrm{L}}$, of sample $\mathrm{B}$ are generally closer to those of sample $\mathrm{C}$. Meanwhile, the best quality of sample B is confirmed with the values of the lowest distribution energy $E_{\mathrm{D}}$.

Conclusions In summary, we have reported the two-component decay of TRPL intensity. The first-decay component was attributed to simultaneous exciton relaxation of free-carrier and localized states; the second-decay one was dominated by localized exciton relaxation. The second-decay lifetime represented the extent of carrier localization or indium aggregation and phase separation. The exciton relaxation lifetime of freecarrier states was related to defect density. We also calibrated the localization energy and distribution energy by fitting our PL and SE data with models. The calibrated results were consistent with those of previous HRTEM and XRD observations, TRPL, and AFM surface scanning.

Acknowledgement This research was supported by National Science Council, The Republic of China (Taiwan), under grants NSC 88-2215-E-002-023, NSC 88-2215-E-002040, NSC 88-2215-E-014-005, and NSC 89-2215-E-002-0036. It was also sponsored by Chung Shan Institute of Science and Technology, Taiwan, R.O.C.

\section{References}

[1] Y. S. Lin, K. J. Ma, C. Hsu, S. W. Feng, Y. C. Cheng, C. C. Liao, C. C. Yang, C. C. Chou, C. M. LeE, and J. I. Chyi, Appl. Phys. Lett. 77, 2988 (2000).

[2] S. Chichibu, T. Azuhata, T. Soda, and S. Nakamura, Appl. Phys. Lett. 69, 4188 (1996).

[3] M. Pophristic, F. H. Long, C. Tran, I. T. Ferguson, and R. F. KarliceK, Jr., J. Appl. Phys. 86, 1114 (1999).

[4] Y. Narukawa, Y. Kawakami, Sz. Fujita, Sg. Fujita, and S. Nakamura, Phys. Rev. B 55, R1938 (1997).

[5] H. S. Kim, R. A. Mair, J. Li, J. Y. Lin, and H.X. JiAng, Appl. Phys. Lett. 76, 1252 (2000).

[6] K. Domen, A. Kuramata, and T. Tanahashi, Appl. Phys. Lett. 72, 1359 (1998).

[7] B. Monemar, Phys. Rev. B 10, 676 (1974).

[8] P. G. Eliseev, P. Perlin, J. Lee, and M. Osinski, Appl. Phys. Lett. 71, 569 (1997). 